\title{
Double Layer Properties and Impedance Characteristics of Uniformly-sized Microporous Carbon from Spent Coffee Grounds
}

\author{
Keisuke Kikuchi $^{1 *}$, Tomohiro Yasue ${ }^{2}$, Rie Yamashita ${ }^{1}$, Satoshi Sakuragawa ${ }^{1}$, Masao Sudoh ${ }^{2}$, \\ Masayuki Itagaki ${ }^{3}$, Tomitaka Toyama ${ }^{4}$ and Suguru Mashimo ${ }^{5}$ \\ 1 Industrial Research Institute of Shizuoka Prefecture, 2078 Makigaya, Aoi-ku, Shizuoka, Shizuoka 421-1298, Japan \\ 2 Graduate School of Engineering, Shizuoka University, 3-5-1 Johoku, Naka-ku, Hamamatsu, Shizuoka, 432-8561, Japan \\ 3 Department of Pure and Applied Chemistry, Faculty of Science and Technology, Tokyo University of Science, 2641 Yamazaki, \\ Noda, Chiba, 278-8510, Japan \\ 4 Mikawa Environmental Technology Co., Ltd., 16 Maeda, Hiroishicho, Gamagori, Aichi, 443-0038, Japan \\ 5 JFE-TEC Co., Ltd., 1 Kawasakicho, Chuo-ku, Chiba, Chiba, 260-0835, Japan \\ * Corresponding author: Fax: 81-54-278-3066, e-mail: k.kikuchi@iri.pref.shizuoka.jp
}

\begin{abstract}
We prepared the spent coffee grounds (SCG) and controlled the steam activation process for them in order to develop SCG-derived activated carbon with uniform micropores that were similar to those of KOH-activated phenolic resin carbon (MSP-20). The SCG-derived activated carbon that we obtained had a high yield, a high specific surface area, and uniform-sized micropores, and it exhibited characteristics different from those of conventional structures containing both micropores and mesopores. The laminated multi-layer EDLC cells achieved a capacitance of higher than $75 \mathrm{~F} / \mathrm{g}$. They were produced with only small variations. The Nyquist plot generated from the impedance measurements showed a fairly vertical rise that can be attributed to the blocking electrode in the low frequency range. This confirms that the pores in the electrodes were used effectively. It was demonstrated that the electric double layer characteristics and impedance characteristics of the SCG capacitors can be changed by controlling the raw material preparation and its activation conditions.
\end{abstract}

Key words: Spent coffee grounds, Activated carbon, Steam activation, Electric double layer capacitor

\section{INTRODUCTION}

An electric double layer capacitor (EDLC) is an energy device that uses physical adsorption and desorption of ions without causing chemical reactions on the electrode surface. As a result, this device is outstanding in terms of rapid charge and discharge and their cycle characteristics. Thanks to these excellent characteristics, EDLCs are being used in more and more important applications, such as acceleration assistance and braking energy regeneration for electric vehicles, as well as power leveling control for renewable energy generation. However, in order for EDLCs to be further developed and promote their widespread use, it is essential that their cost be reduced. Activated carbon is one of the main raw materials used to produce an EDLC, and it is said to account for the bulk of the cost [1]. Recently, demand for activated carbon has been growing rapidly - mainly in developing countries - and the price for activated carbon has continued to rise. This will inevitably have a serious impact on industries in Japan, because Japan has to import most of the activated carbon it uses.

Given the large amounts of spent coffee grounds (SCGs) that are generated in Japan today, we have studied the feasibility of using SCG as a raw material for activated carbon with environmental and economic advantages [2]. Because SCG is inherently porous in structure and contains a lot of carbonaceous compounds (about 51\%), it can be processed to have a large specific surface area, which is the most important property of activated carbon in the production of EDLCs. We have successfully developed excellent SCG-derived activated carbon for EDLCs [3-5]. However, many of our SCG-derived activated carbon samples showed diagonal curves in the low frequency range on the Nyquist plot generated from their impedance measurements, although it would have been desirable for these curves to rise vertically as a result of the behavior of the blocking electrode. This means that EDLCs would have to be used at very low frequencies (or low output power) if all of the surface area of the activated carbon electrodes is to be used to store energy, because the pores of SCG-derived activated carbon vary greatly in size [6].

To improve these characteristics, we meticulously controlled the raw material preparation and steam activation conditions with a view to developing a new SCG-derived activated carbon material with a high yield, a high specific surface area, and uniform porous structure. This newly developed activated carbon was used to fabricate a laminated multi-layer EDLC cell, and electrochemical measurements (constant current charge-discharge tests and impedance measurements) were conducted. As a result, we were able to clarify the relationships between the electric double layer characteristics and the porous structure of the new SCG-derived activated carbon, as well as between the impedance characteristics and the porous structure. 


\section{EXPERIMENTAL}

2.1. Preparation of activated carbon from SCG

SCG is a carbonaceous material, and the type selected for use in this study was one that gives a high yield through a primary carbonization process (this type of SCG is hereinafter referred to as HSCG). It was considered that composition change in the material occurred since HSCG was dried by the fermentation heat of the microorganism. Fig. 1 compares the pyrolysis behavior of HSCG with that of normal SCG (NSCG). Both of them started to thermally decompose at around $250^{\circ} \mathrm{C}$, but their pyrolysis rates progressively deviated when the pyrolysis rate of the HSCG started to decrease at around $300^{\circ} \mathrm{C}$. At the endpoint of $850^{\circ} \mathrm{C}$, NSCG had a yield of $24 \%$, while HSCG had a yield as high as $43 \%$. In the primary carbonization process, approximately 2.5 $\mathrm{kg}$ (bone-dry weight) of HSCG was heated from room temperature to $250^{\circ} \mathrm{C}$ at a heating rate of $6^{\circ} \mathrm{C} / \mathrm{min}$, then to $500^{\circ} \mathrm{C}$ at a rate of $3^{\circ} \mathrm{C} / \mathrm{min}$, and then to $850^{\circ} \mathrm{C}$ at a rate of $6^{\circ} \mathrm{C} / \mathrm{min}$ before being cooled to room temperature in a nitrogen stream. Next, steam activation was performed, with the cooled sample being heated again in a nitrogen stream to $850^{\circ} \mathrm{C}$ (the activation temperature) at a heating rate of $6^{\circ} \mathrm{C}$, at which point the atmosphere was switched to steam with a steam introduction rate of $12 \mathrm{~g} / \mathrm{min}$. This activation process was continued for 10 hours. The steam-activated HSCG carbon that was obtained (hereinafter referred to as Steam-HSCG) was washed with $2 \mathrm{M}$ hydrochloric acid to remove any ash residue and then rinsed thoroughly with distilled water. After that, the Steam-HSCG sample was ground with Multi-Beads Shocker (MB1000, Yasui Kikai Corporation), and measured for particle size distribution. It was also measured for nitrogen adsorption at $77 \mathrm{~K}$ with a gas adsorption instrument (BELSORP-max, BEL JAPAN Inc.) to determine its porous structure.

\subsection{Fabrication of laminated multi-layer EDLC cells}

Arranged according to particle size, the Steam-HSCG samples were mixed with acetylene black (LBG-00966, KISHIDA CHEMICAL Co., Ltd.), carboxymethyl cellulose (WAKO Pure Chemical Industries, Ltd.), and styrene-butadiene rubber (TRP-2000、JSR Corporation) with weight ratios of $85: 10: 3.5: 1.5$, respectively. After that, water was added to prepare a coating liquid with a solid content concentration of $28.8 \%$. Both sides of an etching aluminum foil for collector electrodes were coated with this liquid by using a continuous coating machine (C-type coater manufactured by Thank Metal Co., Ltd.), and then the coated foil was dried at $70^{\circ} \mathrm{C}$ and $120^{\circ} \mathrm{C}$ under normal pressure. It was cut into $\mathrm{A} 4$ size and dried at $120^{\circ} \mathrm{C}$ in a vacuum. After the thickness and coating quantity of the active material layer were measured, the foil was slit into electrodes with a size of 96 by $84 \mathrm{~mm}$ each, and a tab lead was then welded to each of them to make collector electrodes. Twelve electrode films were stacked with separators (TF40, NIPPON KODOSHI CORPORATION) sandwiched between them (11 combinations of positive and negative electrodes) to form a parallel-connected cell. This multi-layered cell was inserted into a laminated bag to be dried again at $60^{\circ} \mathrm{C}$ in a vacuum. An electrolysis solution (1 M TEMA-BF $4 / \mathrm{PC})$ was injected into the bag,

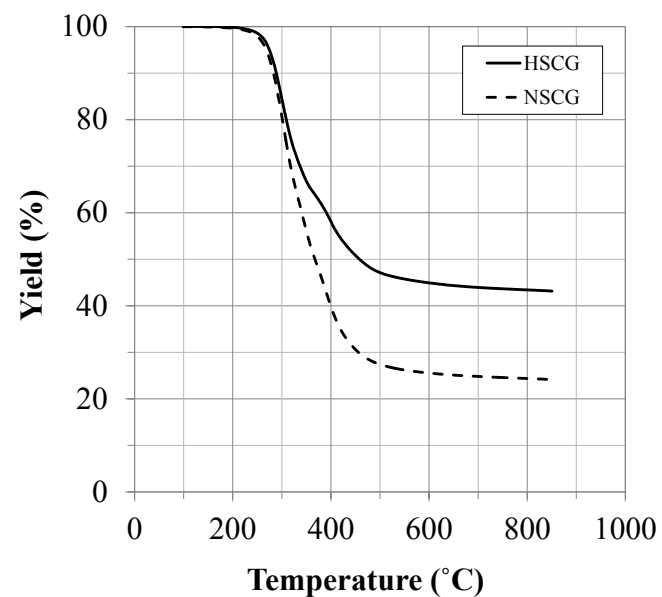

Fig 1. Pyrolysis behavior of SCG carbonaceous material.

Solid line: HSCG, Dashed line: NSCG

and the bag was then heat-sealed with a vacuum sealer. The initial charge-discharge cycles were performed at a voltage of between 0 and $2.5 \mathrm{~V}$ four times with a current density of $0.5 \mathrm{~mA} / \mathrm{cm}^{2}$. After that, the side of the cell was cut open to get rid of generated gas, before being heat-sealed again with a vacuum sealer. Eight cells were fabricated, and a constant current charge-discharge test was conducted on each of them by using a charge-discharge device (SD8 、 HOKUTO DENKO CORPORATION). A current density of $5 \mathrm{~mA} / \mathrm{cm}^{2}$ was used for both the charge and discharge. Each cell was charged from $0 \mathrm{~V}$ to $2.5 \mathrm{~V}$, and then discharged after a one-minute pause. The voltage fall that occurred for 10 ms immediately after the start of the discharge was an IR drop, and the slope of the discharge curve after the IR drop was used together with the following formula to obtain the capacitance of whole cell (C) $[4,7]$.

$$
C=I \times \frac{\Delta t}{\Delta V}
$$

where $\mathrm{I}=$ current $(\mathrm{A}), \mathrm{t}=$ time $(\mathrm{s}), \mathrm{V}=$ voltage $(\mathrm{V})$. Then, the unipolar capacitances per active material weight $(\mathrm{Cw})$ and per active material volume $(\mathrm{Cv})$ were calculated from the following equations.

$$
C w=2 \times C \times \frac{1}{m} \quad, \quad C v=2 \times C \times \frac{1}{v}
$$

where $\mathrm{m}=$ unipolar active material weight $(\mathrm{g}), \mathrm{v}=$ unipolar active material volume (cc).

To examine the impedance characteristics, a potentiostat system (VSP, Bio-Logic) was used to measure the frequency response of the cell within a range of $10 \mathrm{mHz}$ to $1 \mathrm{MHz}$ with a cell voltage of $2 \mathrm{~V}$.

\section{RESULTS AND DI SCUSSION}

3.1 Physical properties of Steam-HSCG

The yield of the activated carbon was $21.2 \mathrm{wt} \%$, and its specific surface area was $1,313 \mathrm{~m}^{2} / \mathrm{g}$. Carbonization was performed with extremely small variations. While the NSCG produced a yield of as low as 8 to $10 \%$ when 


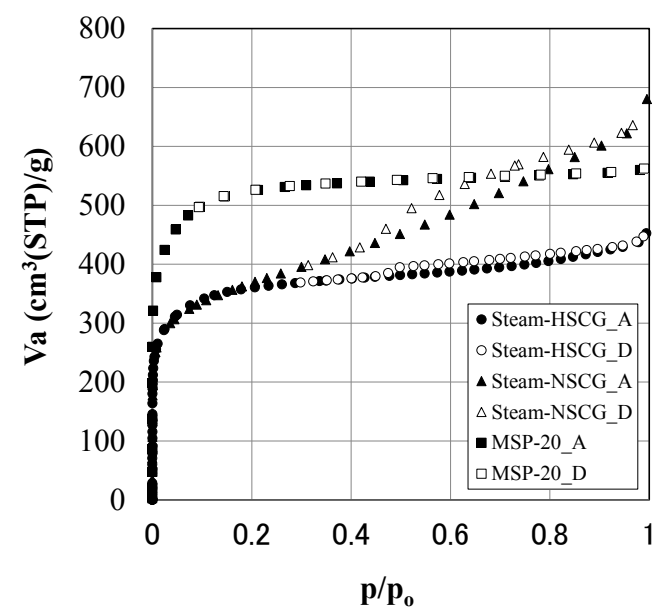

Fig 2. Nitrogen adsorption-desorption isotherm at $77 \mathrm{~K}$.

Circle: HSCG, Triangle: NSCG,

Square: MSP-20

Slid symbol: adsorption, Open symbol: desorption

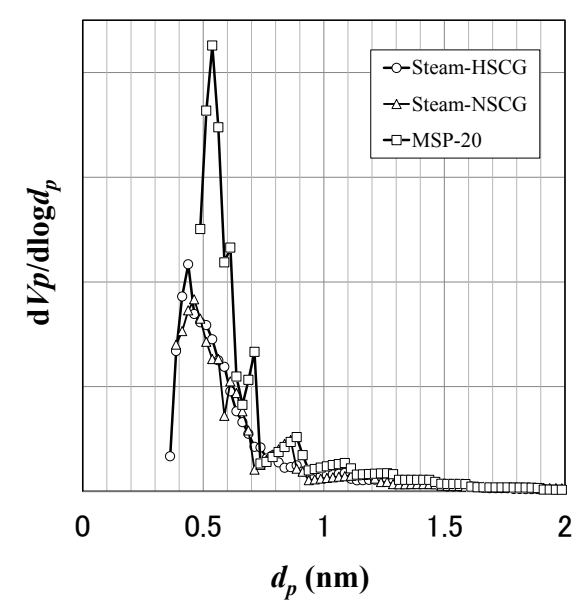

Fig 3. Micropore size distributions analyzed by the HK method.

Circle: HSCG, Triangle: NSCG, Square: MSP-20

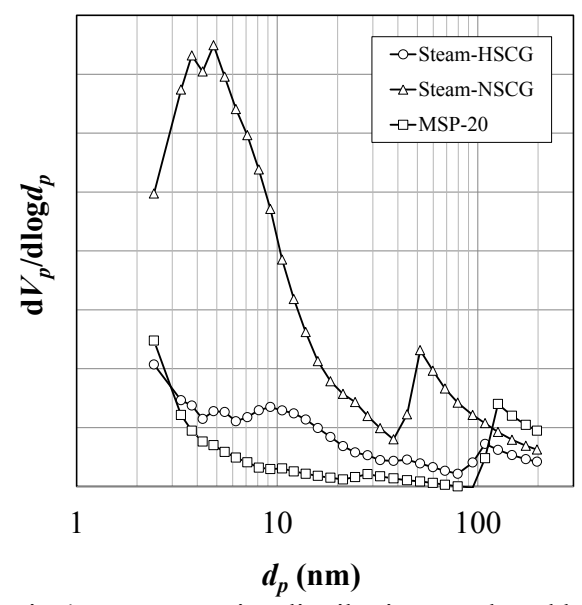

Fig 4. Mesopore size distributions analyzed by the BJH method.

Circle: HSCG, Triangle: NSCG, Square: MSP-20

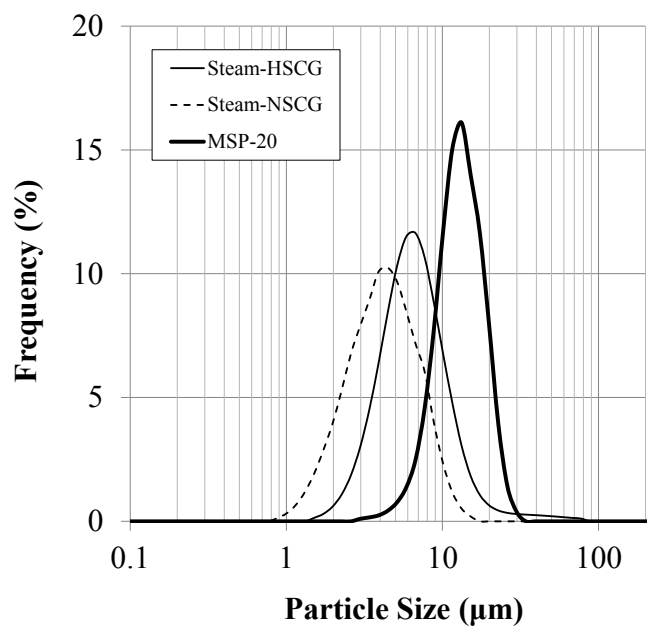

Fig 5. Particle size distributions of each activated carbon.

Thin solid line: HSCG,

Dashed line: NSCG

Thick solid line: MSP-20

a specific surface area of $1,300 \mathrm{~m}^{2} / \mathrm{g}$ was achieved, the HSCG delivered activated carbon with a high yield, as the pyrolysis data suggested it would.

Fig. 2 shows the isotherms for nitrogen adsorption and desorption which is the basis for calculation of the specific surface area and pore size distribution analysis. For comparison, it also shows data for the steam-activated NSCG (Steam-NSCG) sample and $\mathrm{KOH}$-activated phenolic resin carbon (MSP-20). Although it showed a little hysteresis, Steam-HSCG exhibited almost a type I isotherm (characterized by drastic increase in low relative pressure). This implies that it is rich in micropores. Because MSP-20 also exhibited a type I isotherm, it is likely that Steam-HSCG has a porous structure similar to that of MSP-20. In contrast, the Steam-NSCG sample exhibited a type IV isotherm (characterized by increase in middle to high relative pressure and hysteresis), implying it has many mesopores.

Fig. 3 shows the analysis results obtained by using the HK method. The HK method uses averaged potentials inside pores to analyze the pore diameters. This analysis method is suitable for micropores that are free from capillary condensation [8]. Horizontal axis is diameter of the miropore and vertical axis is frequency. All the samples indicated one remarkable peak in micropore range. Steam-HSCG and Steam-NSCG had about the same pore diameter distribution, and their largest peak diameters were $0.44 \mathrm{~nm}$ and $0.46 \mathrm{~nm}$, respectively. MSP-20 had relatively large pore diameters, with a largest peak diameter of $0.54 \mathrm{~nm}$.

Figure 4 shows the analysis results obtained by using the BJH method. The BJH method analyzes the diameters of mesopores based on the Kelvin equation, which expresses the relationship between the pressure and the diameter of a pore with capillary condensation [9]. Horizontal axis is diameter of the mesopore and vertical axis is frequency. Steam-NSCG indicated two remarkable peaks (about $5 \mathrm{~nm}$ and $50 \mathrm{~nm}$ ) unlike in the case of other samples. This analysis revealed that 

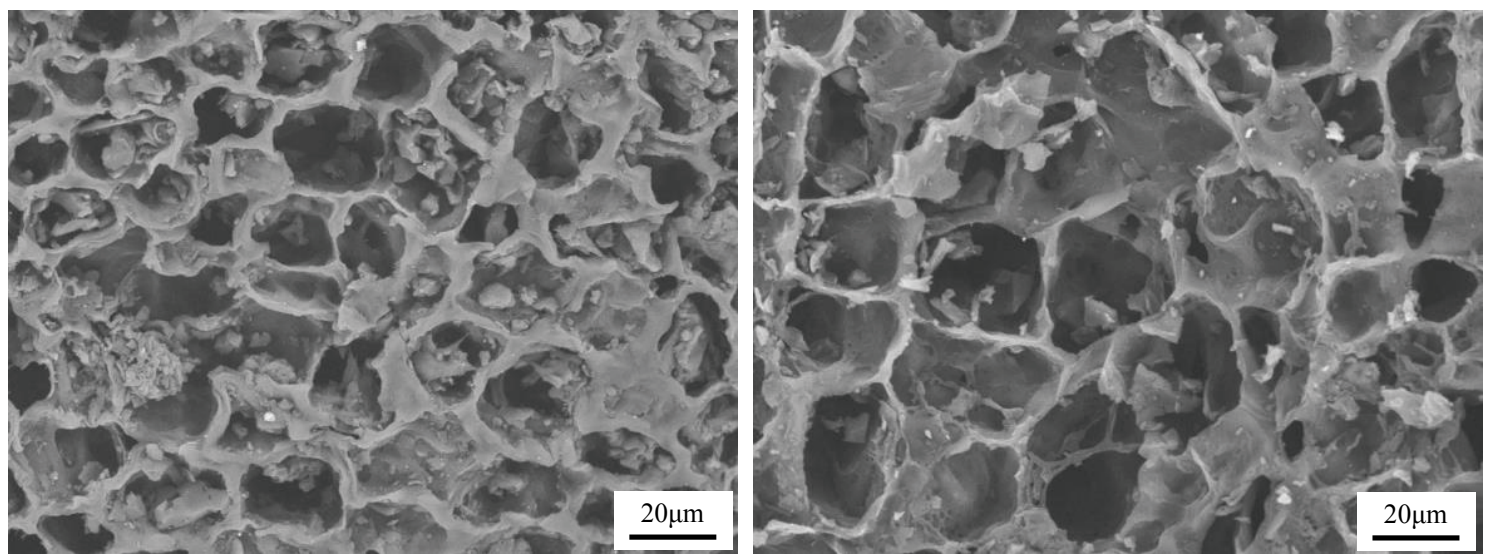

Fig 6. SEM images of Steam-HSCG (left) and Steam-NSCG (right).

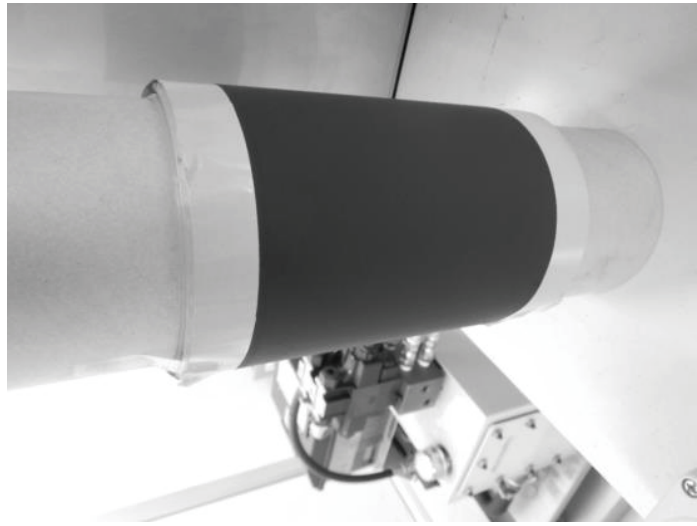

Fig 7. Electrode sheet roll prepared from Steam-HSCG. Coated width: $120 \mathrm{~mm}$

Thickness: $90 \mu \mathrm{m}$

Quantity: $0.49 \mathrm{mg} / \mathrm{cm}^{2}$

Steam-HSCG had considerably fewer mesopores than Steam-NSCG did. MSP-20 had few mesopores, as well.

Based on analysis of the pore diameter distributions, Steam-HSCG was found to have uniform-sized micropores, just like MSP-20 did. In contrast, Steam-NSCG seemed to possess a complex pore structure, in which micopores and mesopores are mixed.

Figure 5 shows the results of particle size distribution measurements. Each sample had a single peak, and MSP-20 had the largest particle sizes, followed by Steam-HSCG and then Steam-NSCG. The median diameters were $12.1,6.0$, and $3.9 \mu \mathrm{m}$, respectively.

Figure 6 shows SEM photographs of Steam-HSCG and Steam-NSCG. The porous structure of the coffee beans was still present even after the activation treatment, and this was probably the reason why these samples were easier to grind than the activated carbon made from phenol resin. Also, Steam-HSCG was relatively harder to grind and had slightly larger particle sizes than Stream-NSCG, probably because Steam-HSCG had a higher yield and a higher density. In any case, the fact that SCG-derived activated carbon is easily ground can be an advantage when manufacturing activated carbon for EDLC electrodes.

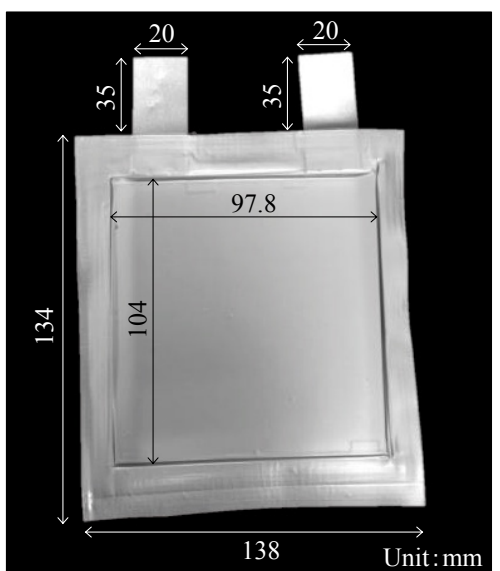

Fig 8 . The appearance and dimensions of the laminated cell fabricated in this study.

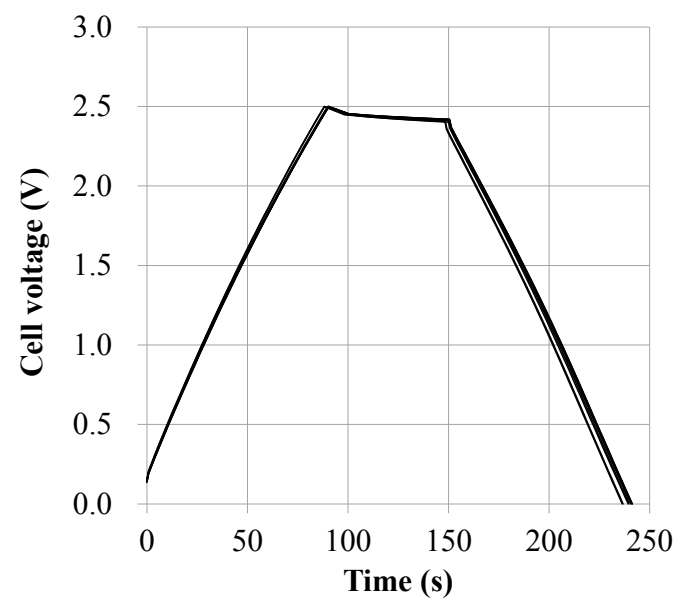

Fig 9. Charge-discharge curves for the eight fabricated cells.

Applied voltage: $0 \mathrm{~V}$ to $2.5 \mathrm{~V}$

Current density: $5 \mathrm{~mA} / \mathrm{cm}^{2}$ 
Table 1. EDLC performance of Steam-SCG. Each value is average of eight laminated cells.

$\mathrm{C}$ : cell capacitance, $\mathrm{Cw}$ : capacitance per active material weight

$\mathrm{Cv}$ : capacitance per active material volume

\begin{tabular}{|c|c|c|c|c|}
\hline & $\mathrm{C}(\mathrm{F})$ & $\mathrm{CW}(\mathrm{F} / \mathrm{g})$ & $\mathrm{Cv}(\mathrm{F} / \mathrm{cc})$ & Internal resistance $(\mathrm{m} \Omega)$ \\
\hline Average & 166.5 & 76.6 & 41.7 & 3.4 \\
\hline Standard deviation & \multicolumn{4}{|c|}{$1.0 \%$} \\
\hline
\end{tabular}

3.2 Electrochemical properties of Steam-HSCG

The thickness and quantity of the active material layer of ground Steam-HSCG that was coated on the electrode sheet were $90 \mu \mathrm{m}$ and $4.9 \mathrm{mg} / \mathrm{cm}^{2}$, respectively. An adequate adhesive force was probably secured, because no part of the layer peeled or ripped off when the sheet was wound up and slit (see Figure 7). Figure 8 shows the appearance and dimensions of the laminated cell fabricated in this study.

Figure 9 shows the charge-discharge curves for the eight fabricated cells. In all the cells, the voltage increased or decreased proportionally over time (or with charge buildup), and they all worked as capacitors.

Table 1 lists the following values obtained from the slopes of the discharge curves and averaged over the eight cells: the cell capacitance (C); the unipolar capacitance per active material weight $(\mathrm{Cw})$; the unipolar capacitance per active material volume $(\mathrm{Cv})$; and the internal resistance calculated from the IR drop. The standard deviations for the capacitance and the internal resistance were $1.0 \%$ and $1.8 \%$, respectively, which shows that these cells were fabricated with small variations. In this study, electrochemical measurements were conducted without applying pressure to the laminated cells. The application of an appropriate amount of pressure would bring the electrodes and the separators into closer contact with each other, thereby reducing the internal resistance.

Figure 10 (a) shows a Nyquist plot for Steam-HSCG that was generated from the Steam-HSCG's AC impedance measurements. In the order of frequency from highest (left side in the figure) to lowest (right side), the plot is divided into bulk resistance, interface resistance (capacitive semicircle), diffusion resistance (upward $45^{\circ}$ slope) and vertical rise of blocking electrode. Steam-HSCG exhibited a clear increase for the blocking electrode in the low frequency range. For comparison, Figure 10 (b) shows Nyquist plots for Steam-NSCG and MSP-20. The measurements were obtained from experimental coin-type cells. MSP-20 shows a clear increase for the blocking electrode in the low frequency range, but only a slight increase for Steam-NSCG. This suggests that Steam-NSCG did not make uniform use of the electrode surface due to its various porous structures, such as micorpores and mesopores [10-12]. In contrast, because Steam-HSCG and MSP-20 had relatively uniform micro-porous structures as can be seen from their pore size distributions, they could make uniform use of the electrode surface and exhibited a vertical rise. As a result, by controlling the raw material preparation and activation processes, we were able to produce (a)

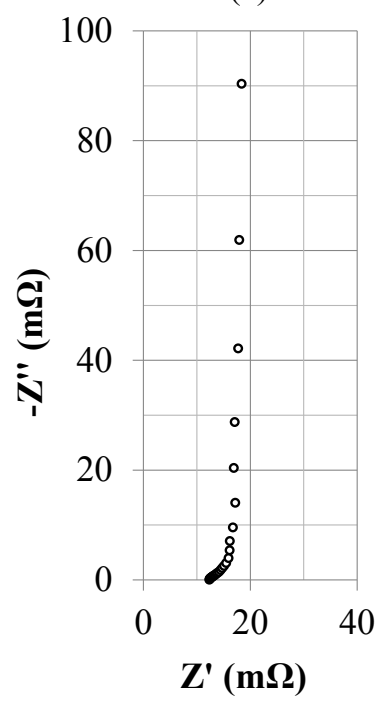

(b)

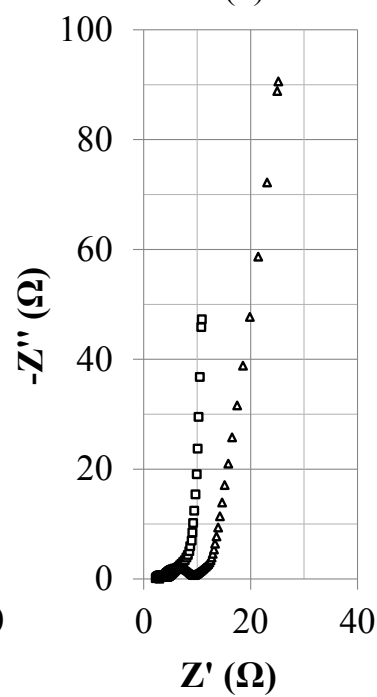

Fig 10. Nyquist plot for each activated carbon.

Circle: HSCG

Triangle: NSCG

Square: MSP20

SCG-derived activated carbon with characteristics similar to those of MSP-20.

\section{CONCLUSION}

We prepared the spent coffee grounds (SCG) and controlled the activation process for them in order to develop SCG-derived activated carbon with uniform micropores that were similar to those of MSP-20. We used steam activation, which is economical and safe, to produce activated carbon on a scale used for practical manufacturing rather than for experiments. The SCG-derived activated carbon that we obtained had a high yield, a high specific surface area, and uniform-sized micropores, and it exhibited characteristics different from those of conventional structures containing both micropores and mesopores. This steam activated SCG carbon was washed and ground, and then mixed with a conduction promoting agent and binder to form a coating liquid. A double-sided coated sheet was produced using a continuous coating method, and this was then used to fabricate laminated cells. The coated layers on the sheet showed good adhesiveness, as they did not peel or rip off. As EDLCs, the cells achieved a capacitance of higher than $75 \mathrm{~F} / \mathrm{g}$. They were produced with only small variations. The Nyquist plot generated from the 
impedance measurements showed a fairly vertical rise that can be attributed to the blocking electrode in the low frequency range. This confirms that the pores in the electrodes were used effectively. It was demonstrated that the electric double layer characteristics and impedance characteristics of the SCG capacitors can be changed by controlling the raw material preparation and its activation conditions. Going forward, this knowledge will be used to establish conditions for manufacturing SCG-derived activated carbon that are tailored to the various specifications for different EDLC applications.

\section{ACKNOWLEDGEMENTS}

This study was performed as a part of policies "New growth strategy research project" of Shizuoka prefecture.

\section{REFERENCES}

[1] Nishino and K. Naoi, Advanced Electrochemical Capacitors: Their Development, Application and Materials II. CMC Publishing Co., Ltd. Tokyo (2007): p.78.)

[2] K. Kikuchi, R. Yamashita and S. Sakuragawa, Trans. Mat. Res. Soc. Japan 37(4): 557-62 (2012).

[3] Keisuke Kikuchi, Tomohiro Yasue, Rie Yamashita, Satoshi Sakuragawa and Masao Sudoh, BIOCOMP2012 $11^{\text {th }}$ Pacific Rim Bio-Based Composites Symposium Proceedings, pp.251-60 (2012)

[4]Keisuke KIKUCHI, Tomohiro YASUE, Rie YAMASHITA, Satoshi SAKURAGAWA, Masao SUDOH and Masayuki ITAGAKI, Electrochemistry, Vol.81, No.10, pp.828-32 (2013)

[5] Keisuke Kikuchi, Tomohiro Yasue, Rie Yamashita, Satoshi Sakuragawa, Masao Sudoh and Masayuki Itagaki, $11^{\mathrm{TH}}$ INTERNATIONAL CONFERENCE ON ECOMATERIALS (ICEM11) Proceedings (2013)

[6] Masayuki Itagaki, Yasunari Hatada, Isao Shitanda and Kunihiko Watanabe, Electrochemica Acta 55 (2010): 6255-62.

[7]S. Shiraishi, Tanso 228: 195-199 (2007).

[8] Horvath, G. and Kawazoe, K. 1983. Method for the Calculation of Effective Pore Size Distribution in Molecular Sieve Carbon. J. Chem. Eng. Japan 16(6): 470-5.

[9] Barrett, P. Elliott, Joyner, G. Leslie and Halenda, P. Paul. 1951. The Determination of Pore Volume and Area Distributions in Porous Substances. I. Computations from Nitrogen Isotherm. J. Amer. Chem. Soc. 73(1): 373-80

[10] H. K. Song, Y. H. Jung, K. H. Lee and L. H. Dao, Electrochim. Acta 44 (1999): 3513-9.

[11] H. K. Song, H. Y. Hwang, K. H. Lee and L. H. Dao, Electrochim. Acta 45 (2000): 2241-57.

[12] H. K. Song, J. H. Sung, Y. H. Jung, K. H. Lee, L. H. Dao, M. H. Kim and H. N. Kim, J. Electrochem. Soc. 151(3) (2004): E102-9.

(Received January 15, 2015; Accepted April 22, 2015) 